\title{
Application research and Prospect of three-dimensional laser scanning technology of Bulk Trade Transfer Metering Technology in Bulk Strategic Materials
}

\author{
Hua dong Hao ${ }^{1, a}$, Hao lei Shi ${ }^{1, b^{*}}$, Cun jun $\mathrm{Li}^{1, \mathrm{c}}$, Xian lei Chen ${ }^{1, \mathrm{~d}}$, and $\mathrm{Yu} \mathrm{He}^{1, \mathrm{e}}$ \\ ${ }^{1} 257$ thousand island road, new town, Zhoushan city, zhejiang province, China \\ Zhoushan Institute of Calibration and Testing for Quality and Technology Supervision \\ agentlehhd@163.com, b181144048@qq.com, licunjun@sina.com, \\ 'zschenxl@163.com, ${ }^{\mathrm{e}}$ 1255936665@qq.com
}

\begin{abstract}
Keywords: bulk strategic materials; bulk cargo metering; draft survey; electronic truck scale measurement; electronic belt measurement; three-dimensional scanning.

Abstract. Bulk strategic materials have important significance to the national economy and national defense. China as a major importer of bulk strategic materials, the accuracy of trade transfer is particularly important. This paper analyzes metering technology development status of bulk strategic materials, including the principle, application scope and influence factors of draft survey, electronic truck scale measurement and electronic belt measurement. Based on spatial three-dimensional scanning technology in the field of application is prospected.
\end{abstract}

\section{Introduction}

Bulk strategic materials have important significance to the national economy and national defense. Depending on the different circumstances in every country, each category of strategic materials is different. In our country, the large import demand of oil, iron ore and some kinds of food are all belong to the Bulk strategic materials commodities. These are energy, raw materials and agricultural products which are indispensable to the steady development of national economy in our country, and they occupy a pivotal position in the import and export trade market. Its trade transfer measurement accuracy is particularly important, how to improve the measurement accuracy of bulk, reduce trade disputes and create economic benefits, establish measurement image and enhance the credibility of measurement has important practical significance and far-reaching influence. According to the above problems, this paper discusses the development and prospect of bulk trade transfer metering technology in bulk strategic materials.

\section{The development status of bulk trade transfer metering technology in bulk strategic materials}

Currently, there are three measurement methods commonly used in bulk strategic materials: draft survey, electronic truck scale measurement, electronic belt measurement. The following is an introduction to the above three methods.

\section{Draft Survey}

Draft Survey is a cargo weight method commonly used in low-value bulk cargo shipping. Due to its high speed, low cost and has a certain scientific, it has been widely recognized and applied in international trade. Ship draft survey is based on "Archimedes' principle", by separately measure the ship's draft and ship fuel, fresh water and ballast water stocks around twice of cargo before and after loading or unloading, simultaneous determination of ship outboard water density, and then according to the dead weight scale, displacement scale as well as water tanks, oil tanks' meters and correction table and other relevant information of marine charts to calculate the weight of the ship's cargo ${ }^{[1]}$. 
Suppose the ship displacement before loading (or after unloading) is $\triangle \mathrm{f}$, ship fuel, fresh water, pressure water and other reserves total are Gf, ship displacement after loading (or before unloading) is $\triangle \mathrm{a}$, for the total reserves of the whole ship are Ga, the cargo loading (or unloading) can be calculated by formula (1):

$$
Q=\left(\Delta_{a}-G_{a}\right)-\left(\Delta_{f}-G_{f}\right) .
$$

This method does not use the smaller or the invariant amount of before loading or unloading of the ship, such as ship's constant, lightship weight, the weight of crew, baggage, spare parts, grains etc, so the calculation is more accurate ${ }^{[2]}$. The factors which influence the accuracy of the measurement are mainly the size of the wind waves, the ship's longitudinal and transverse inclination, the change of the material on the ship, the ballast water, the density of water in the harbor and the density of ballast water, etc. General draft survey errors can be controlled within $0.5 \%$. Since the draft survey accuracy is lower than other measurement methods, and therefore only suitable for low value bulk cargoes, such as ore, coal, fertilizer, pig iron, etc.

\section{Electronic truck scale measurement}

Truck scale commonly known as weighbridge with two types of analog and digital, it is the main weighing equipment for bulk cargo measurement. Before the 1980s truck scale commonly is mechanical, which is use of lever principle pure mechanical structure, also known as mechanical weighbridge. In the middle period of 1980 s, with the high precision weighing sensor technology is becoming more and more mature, the mechanical weighbridge is gradually replaced by high precision, good stability and convenient operation of electronic truck scale. Electronic truck scale is mainly composed of bearing device, weighing display instrument, weighing sensors, fittings, limit switch and junction box and other components, it can also be equipped with printers, display screens, computers and weighing management software and regulated power supply and other external equipment.

The working principle of electronic truck scale is put the loading car arrange on the scale platform, under the action of gravity, the scale platform transfer gravity to the weighing sensor, so that the weighing sensor elastic body deformation, paste in the elastic strain of the beam on the strain gauge bridge loses balance, output and quality value proportional to the electrical signal, by linear amplifier to amplify the signal, then after A/D conversion to digital signal and by the microprocessor(CPU) process the quality signal, then directly display the quality value, transfer the weighing data to the computer management system for processing ${ }^{[3]}$. The errors of electronic truck scale measurement accuracy have weighing error, discernment error, repeatability error, etc.

\section{Electronic belt measurement}

Electronic belt scale is a general term of electronic belt scale, quantitative feeder, hanging belt scales. In general, the belt scale is a separate weighing device which is installed on the long conveyor belt rack. It only materials through cumulative weighing on the conveyor belt, don't control the size of material flow. Quantitative feeder is composed of endless belt, load receptor, motor, weighing and speed sensor, which is set of weighing measurement and flow control in one of the continuous weighing equipment, also called speed scale. Hanging scale is a continuous weighing device hanging up whole scale with weighing sensor (including an endless belt, load receptor, motor, sensor etc), it features the whole hanging is not affect by other factors, so the measurement accuracy is high, but also can according to the flow rate to control the speed of feeding device in order to achieve the purpose of quantitative feeding.

Electronic belt scale weighing bridge install on the conveyor frame, when the material through measuring roller belt material weight was detected in leverage in the weighing sensor, generates a signal is proportional to the belt load voltage. The speed sensor is directly connected to a large diameter measuring roller, and provides a series of pulses, each of which is represented by a belt moving unit, and the frequency of the pulse is proportional to the speed of the belt. The weighing instrument receives signals from the weighing sensor and the speed sensor, and obtains an instantaneous flow value and the accumulated weight value through the integral operation. Effect of 
electronic belt measurement accuracy error factors are calibration errors and environmental errors. If the condition environment is better, the general error of the environment is less than $0.05 \%$.

\section{Analysis technique status}

At present, these three kinds of methods in the application and the accuracy each has its own defects, mainly manifest in: (1) Draft Survey affected by the storm, hydrological and other factors, it can't guarantee the accuracy. (2) Although the investment is small for truck scale, but the operation process is complex, must after empty car measurement, loading, full vehicle measurement, unloading process, the measurement process exist high energy consumption, low operating efficiency, the car back and forth is likely to cause confusion and measurement errors. (3) Although electronic belt scale using a pressure sensor, small investment, less land occupation, easy to use, bur its structure is complex, difficult to adjust, easy to deformation, lower stability, calibration must be carried out regularly. Calibration of belt scale is a very complicated work, according to the regulations for the national metrological verification regulations JJG195-2002 belt scale verification regulation, the best way to calibrate the belt scale is physical verification. But it is difficult to manufacture large scale physical verification device.

\section{The prospect of bulk cargo transfer measurement technology in bulk strategic materials}

\section{The prospect of new method}

With the development of science and the progress of the times, Especially the modern computer technology and communication technology is widely used in the field of measurement, making the development of measurement technology by leaps and bounds. The three-dimensional scanning is a technology that integrates light, machine, electricity and computer technology. This technique is often used to scan the shape, structure and color of the object, and get the three-dimensional profile data of the object. It overcomes the limitations of the traditional measurement techniques, by using non-contact active survey, the technique can directly capture the high precision three-dimensional data of the object. It can scan any object, quickly convert the information of the real world to the data that can be processed. It has been successfully applied in the field of surveying and mapping engineering, structural measurement, construction, historic site measurement, emergency service, entertainment and so on ${ }^{[4]}$. There are three kinds of methods to obtain three-dimensional data:

(1)The method of structured light: The basic idea of this method is to obtain the depth information of the scene using the geometric information of the structure light projection. With a light source having a specific structure shapes projected onto the object to be measured, forming a light stripe, then the object captured by the camera, based on the relative position of the light source and the camera, according to the theory of computer vision, the three-dimensional coordinates of irradiation point can be calculated from the shape of light stripe.

(2)The method of pulse ranging: The method use the rangefinder active transmitter detection signal to the surface of object, signal encounter object surface reflect back, according to the flight time or phase change of the signal, the flight distance of the signal can be calculated, so then we can obtain the spatial position information of the object surface.

(3)The method of phase ranging: This method is to use the frequency of radio spectrum, by amplitude modulation of laser beam, and determination the phase retardation generated by modulated light back and forth surveying line once, then according to the wavelength of the modulated light, conversion of the distance represented by the phase delay, that is use indirect method to measure the time required for light back and forth surveying line.

\section{The new method research status}

The three-dimensional laser scanning technology for domestic use in the measurement of bulk cargo has been carried out for some studies. In the aspect of grain management, in 2009, the professor of Capital Normal University Zhang Aiwu applied the technique to the national grain stockpile inventory work and carried out the research of non-contact granary reserves rapid detection technology ${ }^{[5]}$. In 2012, the Jilin Province Grain and Oil Research and Design Institute of Zhu Tiejun 
and other people used the technology to carry out a preliminary test in the irregular shape of the grain bulk volume measurement, the results show that the technique calculation the amount of food is feasible and has stronger practicability. The technique can be applied to the amount of grain out of storage statistics, inventory grain regulatory network system in grain storage enterprises ${ }^{[6]}$. In the aspect of coal yard management, due to accuracy of the thermal power plant coal storage capacity measurement results directly affect the thermal power plant coal consumption rate and economic operation index, professor Zhou Jianxun Nanjing University of Science and Technology, professor Fan Huimin Xi'an Technological University, They were to apply the technology to build inventory and the amount of coal storage "digital coal yard" work, and developed the coal field coal pile automatic metering system ${ }^{[7]}$. In this paper, the author also used a $3 \mathrm{D}$ scanner to scan and data processing of coal pile (Figure 1). But at present, the calculation speed and accuracy for the volume of coal piles can not be taking into account at the same time and can't achieve the dynamic three-dimensional reconstruction of coal pile, nor can real-time display the coal pile usage. For large open-air coal pile, unable to effectively multi-site registration, and because of heavy dust, high temperature, electromagnetic interference, moisture condensation and other adverse factors may affect the measurement accuracy and the working stability. It is necessary to design automatic temperature control system in order to provide a suitable environment for the operating temperature of the laser scanner, and use dustproof sealing device to protect the scanner to avoid dust dirty lens.

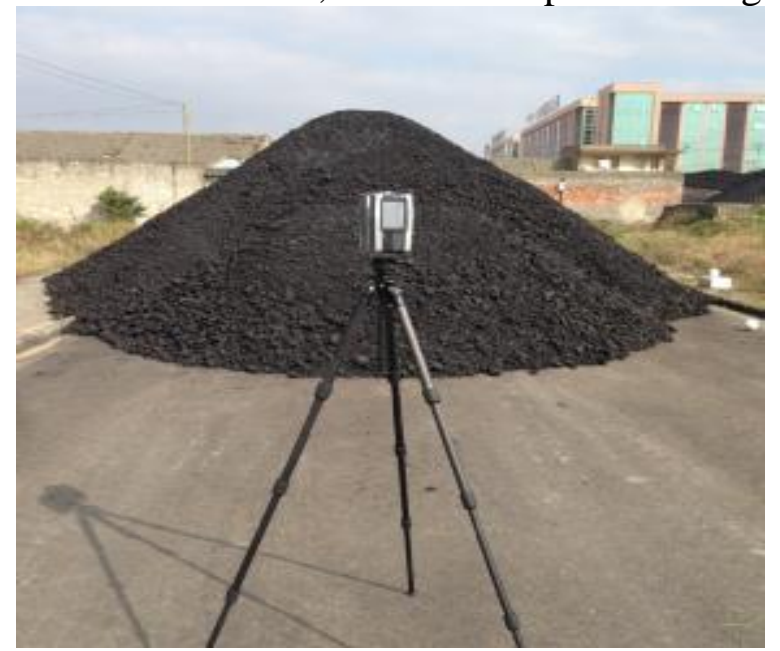

Fig. 1 3D scanner was used to scan coal pile

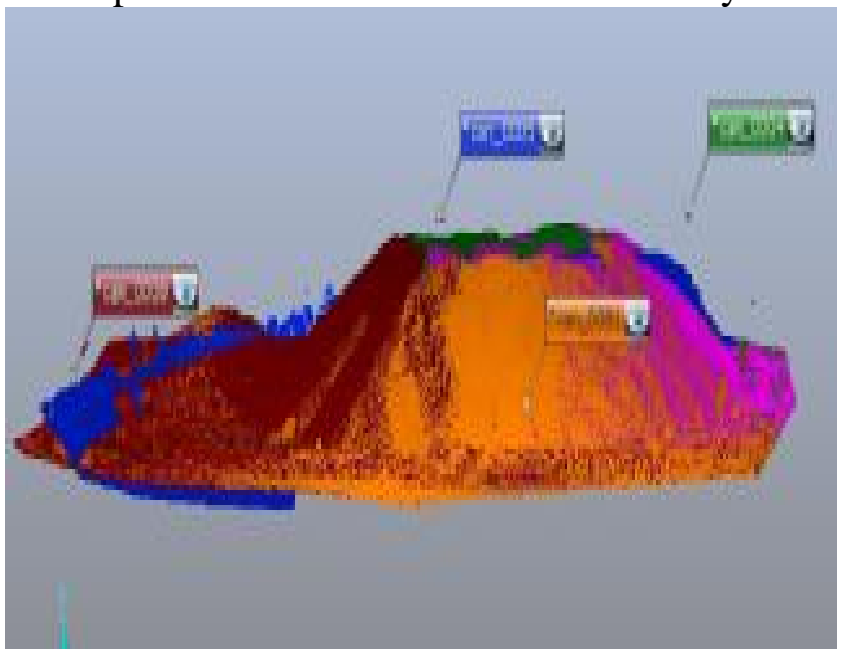

Fig. 2 3D scanning processing of coal pile

\section{Summary}

In this paper, according to current situation of bulk strategic materials measurement technology, including the measuring principle, scope and influence factors of draft survey, electronic truck scale measurement and electronic belt measurement were analyzed. Meanwhile, based on spatial three-dimensional scanning technology in the field of application is prospected. If the three-dimensional laser scanning technology can be applied to the bulk strategic materials in bulk-cargo measurement work will significantly improve the efficiency and accuracy of the measurement, it will be a major breakthrough in the field of bulk-cargo measurement, become a useful supplement for bulk measurement standards in our country, it has important theoretical significance and application value.

\section{Acknowledgment}

The research is supported by the Research Project of science and technology of Zhoushan city (2013C31049and2015C31043) and Project of Quality Supervision System of Zhejiang province(20150266). The authors would like to express a special thank for its support. 


\section{Reference}

[1] Qiu Xinyong, Talk about the draft check for general cargo ship, J. Navigation Technology. 6(2005)25.

[2] Zhang Gang, The principles and methods of draft survey in bulk-cargo transportation, J. China Navigation. 69(2006)35-38.

[3] Fang Yuanbai, The principle and application of electronic belt scale, Beijing, 1994.

[4] Zhang Qifu, Sun Xianshen, Three dimensional laser scanner measurement method and Prospect, J. Beijing Surveying and Mapping. 1(2011)39-42.

[5] Yang Fan, Zhang Aiwu, Dai Yucheng, Application of three-dimensional laser scanning technology in granary reserve detection, J. Chinese Journal of Lasers, 37(2010)317-323.

[6] Zhu Tiejun, Study on measuring grain bulk by laser scanning, J. Grain Science and Technology and Economy, 37(2012)30-31.

[7] Xiong Wentao, Automatic measuring system of coal heaps volume, Nanjing, Nanjing University of Science and Technology, 2002. 